\title{
On the Cocircuit Graph of an Oriented Matroid*
}

\author{
R. Cordovil, ${ }^{1}$ K. Fukuda, ${ }^{2}$ and A. Guedes de Oliveira ${ }^{3}$ \\ ${ }^{1}$ Departamento de Matemática, Instituto Superior Técnico, \\ Av. Rovisco Pais, P-1049-001 Lisboa, Portugal \\ cordovil@math.ist.utl.pt \\ ${ }^{2}$ Institute for Operations Research, ETHZ, \\ CH-8092 Zurich, Switzerland \\ and \\ Department of Mathematics, EPFL, \\ CH-1015 Lausanne, Switzerland \\ fukuda@ifor.math.ethz.ch \\ ${ }^{3}$ Centro de Matemática do Porto, Faculdade de Ciências U. Porto, \\ Praça Gomes Teixeira, P-4050 Porto, Portugal \\ agoliv@fc.up.pt
}

\begin{abstract}
In this paper we consider the cocircuit graph $\mathrm{G}_{\mathcal{M}}$ of an oriented matroid $\mathcal{M}$, the 1 -skeleton of the cell complex $\mathcal{W}$ formed by the span of the cocircuits of $\mathcal{M}$. In general, $\mathcal{W}$ is not determined by $\mathrm{G}_{\mathcal{M}}$. However, we show that if the vertex set (resp. edge set) of $\mathrm{G}_{\mathcal{M}}$ is properly labeled by the hyperplanes (resp. colines) of $\mathcal{M}, \mathrm{G}_{\mathcal{M}}$ determines $\mathcal{W}$. Also we prove that, when $\mathcal{M}$ is uniform, the cocircuit graph together with all antipodal pairs of vertices being marked determines $\mathcal{W}$. These results can be considered as variations of Blind-Mani's theorem that says the 1-skeleton of a simple convex polytope determines its face lattice.
\end{abstract}

\section{Introduction and Notation}

Let $E$ be a finite set of vectors of $\mathbb{R}^{d+1}$ and, for each vector $e \in E$, consider the intersection (a $(d-1)$-dimensional sphere) of the hyperplane perpendicular to $e$ through the origin with the unit sphere $S^{d}$. The cell decomposition of the sphere thus obtained

\footnotetext{
* The first author's research was partially supported by FCT and PRAXIS XXI through the Research Units Pluriannual Funding Program, and by the projects PRAXIS/2/2.1/MAT/125/94 and PRAXIS/PCEX/C/MAT/39/96. The third author's research was partially supported by FCT and PRAXIS XXI through the Research Units Pluriannual Funding Program, and by the project AGC/PRAXIS XXI/2/2.1/MAT/63/94.
} 
does not change when vectors are replaced by nonzero scalar multiples. Note that in this cell complex the normal vectors of the hyperplanes passing through a 0-cell span a linear hyperplane in $\mathbb{R}^{d+1}$, and those associated with a 1 -cell span a coline (i.e., $(d-1)$-space).

More generally, given the Folkman-Lawrence representation of an oriented matroid $\mathcal{M}$ on a finite set $E$ as an arrangement of pseudospheres indexed by $E$ in $S^{d}$, the cell complex $\mathcal{W}$ is combinatorially represented by the span of the cocircuits of $\mathcal{M}$. In general (see Example 2.1), the 1-skeleton of $\mathcal{W}$ (the cocircuit graph $\mathrm{G}_{\mathcal{M}}$ of $\mathcal{M}$ ) does not determine $\mathcal{W}$. However, we prove that it does determine $\mathcal{W}$ if the vertices (resp. edges) of the graph are labeled by the corresponding subset of $E$.

This result can be improved in the rank 3 and in the uniform case under the condition that the opposite pairs of vertices are marked. Then, as an application of the previous statement, Theorem 2.3, we prove that the graph is by itself sufficient to determine the complex.

The results presented here are also closely related to the work of Roudneff in [13]. In particular Theorem 2.3 is a generalization by duality of Theorem 3.1 in [13], and the two theorems coincide in the uniform case. Applied to the rank 3 case, our main result implies as well a theorem of Goodman and Pollack, namely, Theorem 2.9 of [8]. It should be mentioned that our results can be considered as variations of Blind-Mani's theorem [4] that says the 1-skeleton of a simple convex polytope determines its face lattice (see also [11]). When an oriented matroid is representable, the complex $\mathcal{W}$ coincides with the face lattice of the dual of a $(d+1)$-zonotope. Thus our results show how theorems of Blind-Mani's type can be phrased for a certain class of nonsimple polytopes.

For a long time our results remain unpublished, although they have been used more than once, mainly for algorithmic purposes. Recently, they were also the base for the method of proof of the main result of [5].

We suppose the reader is familiar with the basic terminology most commonly used in matroid theory and in oriented matroid theory, like those presented [2], [14], and [15].

Let us fix some notations we use. We use $E$ to denote the set $\{1,2, \ldots, n\}$ of $n$ elements, which is also denoted by $[n]$. For an element $C$ of $\{-, 0,+\}^{E}$, we consider as usual the positive support $C^{+}:=\left\{e \in E: C_{e}=+\right\} \subset E$, and similarly the zero support $C^{0}$ and the negative support $C^{-}$. The support of $C$ is $\underline{C}:=C^{+} \cup C^{-}$. For any subset $A$ of $E$, by reversing the signs of all the coordinates of $C$ indexed by $A$, we obtain ${ }_{\bar{A}} C$. Given an oriented matroid $\mathcal{M}$ (over $E$ ), with set of (signed) cocircuits $\mathcal{C}$ and a subset $A$ of $E$, the set of form ${ }_{\bar{A}} \mathcal{C}:=\left\{{ }_{\bar{A}} C \mid C \in \mathcal{C}\right\}$ is the set of cocircuits of a reorientation of $\mathcal{M}$. We represent the underlying nonoriented matroid by $\underline{\mathcal{M}}$, whose (nonoriented) cocircuits are merely the supports of cocircuits of $\mathcal{M}$. We denote the collection of all possible reorientations of $\mathcal{M}$ by $\mathrm{OC}(\mathcal{M})$, called the reorientation class of $\mathcal{M}$. Two oriented matroids $\mathcal{M}$ on $E$ and $\mathcal{M}^{\prime}$ on $E^{\prime}$ are said to be isomorphic if there exists a bijection $f$ from $E$ to $E^{\prime}$ such that $\mathrm{OC}(f(\mathcal{M}))=\mathrm{OC}\left(\mathcal{M}^{\prime}\right)$, where $f(\mathcal{M})$ denotes the oriented matroid on $E^{\prime}$ obtained from $\mathcal{M}$ by the map $f$. The isomorphism class of all oriented matroids isomorphic to an oriented matroid $\mathcal{M}$ is denoted by $\operatorname{IC}(\mathcal{M})$.

As usual, we consider the set $\mathcal{W}$ of covectors or the span of cocircuits of $\mathcal{M}$, as the compositions of the signed cocircuits (see p. 141 of [2]), defined by $(X \circ Y)^{ \pm}=$ $X^{ \pm} \cup\left(Y^{ \pm} \backslash X^{\mp}\right)$. The partial order defined by the relation

$$
X \leq Y \quad \Longleftrightarrow \quad \text { for every } e \in E, Y_{e}=X_{e} \text { if } X_{e} \neq 0
$$


captures the natural facial incidence relation among cells of the associated arrangement of pseudospheres.

By the Folkman-Lawrence representation theorem [2], we may think of $\mathcal{W}$ as a cell complex of cells of form

$$
[X]=\{Y \in \mathcal{W} \mid Y \leq X\} .
$$

The topological dimension of $[X]$ is one less than the corank of $X^{0}$, a flat of $\mathcal{M} . \mathcal{W}$ is a piecewise linear sphere of $\operatorname{dimension} \operatorname{rank}(\mathcal{M})-1$ and the topes, i.e., the cells of maximal dimension, are in one-to-one correspondence with the acyclic reorientations of $\mathcal{M}$.

The abstract cell complex $\mathcal{W}$ determines the isomorphism class of $\mathcal{M}$. The span $\mathcal{W}^{\prime}$ of cocircuits of $\mathcal{M}^{\prime}$ is isomorphic to $\mathcal{W}$ if and only if $\mathcal{M}^{\prime}$ is isomorphic to $\mathcal{M}$. Indeed, it is possible to reconstruct the isomorphism class of $\mathcal{M}$ from the top two levels of the complex, the tope graph, whose vertices are the topes and whose edges are the cells of dimension one less (see [1] and [6]). For a polynomial algorithm for the reconstruction, see [7]. The present paper studies how much information the bottom two levels of the complex contain.

Definition 1.1. We call the 1-skeleton of the cell complex $\mathcal{W}$ associated with $\mathcal{M}$ the cocircuit graph $\mathrm{G}_{\mathcal{M}}$ of $\mathcal{M}$.

Hence, $\mathrm{G}_{\mathcal{M}}$ consists of:

(i) the set of vertices $\mathrm{VG}_{\mathcal{M}}$ formed by the signed cocircuits of $\mathcal{M}$;

(ii) the set of edges $\mathrm{EG}_{\mathcal{M}}$ of pairs of form $\{V, W\} \subset \mathrm{VG}_{\mathcal{M}}$ for which there exists $X \in \mathcal{W}, X \geq V, W$, such that $X$ covers $V$ (and hence $W$, by the diamond property [3]): $X=V \circ W=W \circ V$; the set complement of its support, $X^{0}$, is a coline of $\underline{\mathcal{M}}$.

Let $\mathcal{M}$ be an oriented matroid of rank 2. It is easy to see that its cocircuit graph $\mathrm{G}_{\mathcal{M}}$ can be seen as an even-length cycle $\left(C_{1}, C_{2}, \ldots, C_{s},-C_{1},-C_{2}, \ldots,-C_{s}\right)$ of cocircuits such that any two consecutive ones are adjacent. Its reorientation class $\mathrm{OC}(\mathcal{M})$ is thus representable as the cyclic reordering of the sequence $\left(H_{1}, H_{2}, \ldots, H_{s}\right)$ where $H_{j}:=C_{j}^{0}$, the hyperplane associated with $C_{j}$.

It should be noted that a cocircuit graph is merely a graph, represented as abstract sets of vertices and edges which do not carry any explicit information about the original sign vectors.

\section{Main Theorem}

We note first that the isomorphism class of an oriented matroid $\mathcal{M}$ is not always determined by the cocircuit graph $\mathrm{G}_{\mathcal{M}}$ :

Example 2.1. Let $\mathcal{M}$ and $\mathcal{M}^{\prime}$ be the affine oriented matroids of the point configurations in Fig. 2.1, where we have the vertices of a tetrahedron together with three extra points interior to three different edges, forming a triangle parallel to a base, in the first case, 

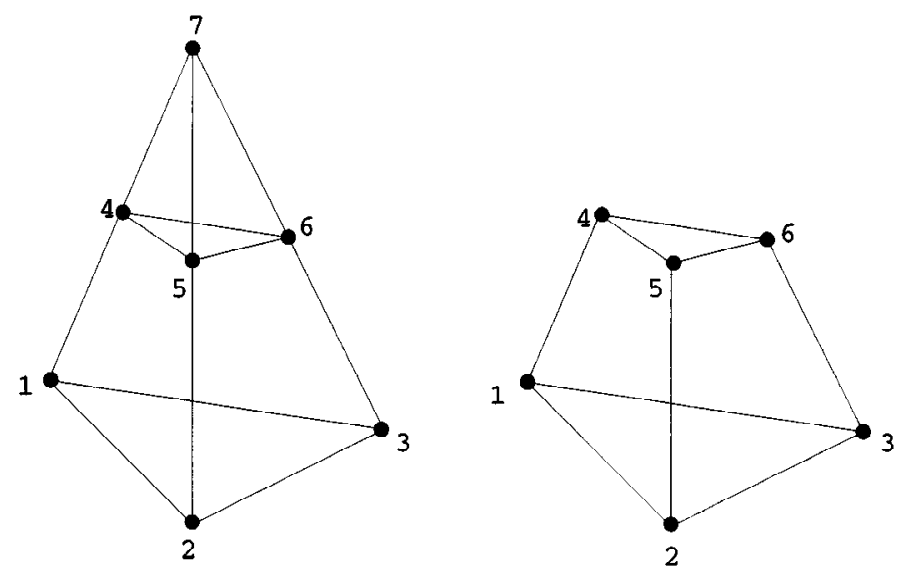

Fig. 2.1. Two configurations whose cocircuit graphs are identical.

and the same without the vertex opposite to the base. It is clear that two configurations generate the same set of linear hyperplanes (i.e. planes). There are exactly two cocircuits associated with each hyperplane, representing the partition of the points not on the hyperplane. One can easily verify that the extra point 7 of the left has no effect on the adjacency of cocircuits, and thus $\mathrm{G}_{\mathcal{M}}$ and $\mathrm{G}_{\mathcal{M}^{\prime}}$ are isomorphic, although clearly $\mathcal{M}^{\prime}$ is not isomorphic to $\mathcal{M}$.

We show that this is not the case when the graph $\mathrm{G}_{\mathcal{M}}$ is properly labeled. More precisely, we consider:

Definition 2.2. We say the cocircuit graph $\mathrm{G}_{\mathcal{M}}$ is vertex-labeled if every vertex $C \in$ $\mathrm{VG}_{\mathcal{M}}$ is labeled by its zero support, i.e., the hyperplane $C^{0}=E \backslash \underline{C} . \mathrm{G}_{\mathcal{M}}$ is edge-labeled if every edge $\{C, D\} \in \mathrm{EG}_{\mathcal{M}}$ is labeled by its zero support, i.e., the coline $W^{0}=E \backslash \underline{W}$, for $W=C \circ D$.

Then we can state the first main theorem:

Theorem 2.3. Let $\mathcal{M}$ be a simple oriented matroid, of rank greater than or equal to 2. Then any one of the following structures determines all of them:

(a) $\mathrm{OC}(\mathcal{M})$;

(b) the vertex-labeled graph $\mathrm{G}_{\mathcal{M}}$;

(c) the edge-labeled graph $\mathrm{G}_{\mathcal{M}}$;

(d) the (nonoriented) matroid $\underline{\mathcal{M}}$, together with the classes $\mathrm{OC}(\mathcal{M} / L)$ for all colines $L$ of $\underline{\mathcal{M}}$.

Proof. Note that when $\operatorname{rank}(\mathcal{M})=2$, clearly the structures in (a), (b), and (d) provide the information for each other. Furthermore, the structures in (b) and (c) are equivalent, in that vertex- and edge-labeling clearly determine each other. On the other hand, knowledge 
of (a) implies knowledge of (b), according to the definitions. Hence, we start by proving that knowledge of (b) $(\Longleftrightarrow$ (c)) implies that of (d):

Suppose that we know the vertex-labeled graph $\mathrm{G}_{\mathcal{M}}$. Then $\underline{\mathcal{M}}$ is determined by the labels. For a coline $L$ of this matroid, consider the subgraph of $\mathrm{G}_{\mathcal{M}}$ of edges labeled by $L$. This new graph is, by definition, the cocircuit graph $\mathrm{G}_{\mathcal{M} / L}$ of $\mathcal{M} / L$. Since this new oriented matroid has rank 2 , its graph is a simple cycle, and thus the reorientation class is represented by the subgraph of the graph $\mathrm{G}_{\mathcal{M}}$ whose edge labels contain $H \backslash L$.

Now, we prove that, given, as in (d), the underlying matroid $\mathcal{M}$ of $\mathcal{M}$ and the reorientation classes $\mathrm{OC}(\mathcal{M} / L)$ for all colines $L$ of $\underline{\mathcal{M}}$, the reorientation class of $\mathcal{M}$ is uniquely determined. More precisely, we prove by induction on $n$ the statement

(*) if an oriented matroid $\mathcal{M}^{\prime}$ satisfies $\underline{\mathcal{M}^{\prime}}=\underline{\mathcal{M}}$ and $\mathcal{M}^{\prime} / L \in \mathrm{OC}(\mathcal{M} / L)$ for every coline $L$ of $\underline{\mathcal{M}}$, then $\mathrm{OC}\left(\mathcal{M}^{\prime}\right)=\mathrm{OC}(\mathcal{M})$.

Clearly $(*)$ is true if $\operatorname{rank}(\mathcal{M})=2$. In addition, $(*)$ is true if $\operatorname{rank}(\mathcal{M})=n$, because then every element $e \in E$ is a coloop and clearly $\mathcal{M}$ has just one orientation.

Let $\mathcal{M}$ be an oriented matroid on $E=[n]$ with rank $r>2$ and $n>r$. By induction hypothesis, we assume that the statement $(*)$ is true for any oriented matroid of the same rank and a smaller number of elements. Now suppose that an oriented matroid $\mathcal{M}^{\prime}$ satisfies $\underline{\mathcal{M}^{\prime}}=\underline{\mathcal{M}}$ and $\mathcal{M}^{\prime} / L \in \mathrm{OC}(\mathcal{M} / L)$ for every coline $L$ of $\underline{\mathcal{M}}$. We will show $\mathcal{M}^{\prime} \in \overline{\mathrm{OC}}(\mathcal{M})$. For this, we may reorient $\mathcal{M}^{\prime}$ as we wish for the proof below.

Since $n>r, \underline{\mathcal{M}}$ contains an element which is not a coloop. By renumbering if necessary, we may suppose $n$ is not a coloop. Thus $\mathcal{M}^{\prime}([n-1])$ has rank $r$ as well. Since knowledge of (d) for $\mathcal{M}$ clearly induces knowledge of (d) for $\mathcal{M} \backslash n$, and by the induction hypothesis, $\mathrm{OC}\left(\mathcal{M}^{\prime}([n-1])\right)=\mathrm{OC}(\mathcal{M}([n-1]))$. By reorientation of $\mathcal{M}^{\prime}$ if necessary, we may assume $\mathcal{M}^{\prime}([n-1])=\mathcal{M}([n-1])$. Then the following statement is valid:

(d') Given a coline $L$ of $\underline{\mathcal{M}}$ such that $n \notin L$, either $\mathcal{M} / L=\mathcal{M}^{\prime} / L$ or $\mathcal{M} / L=$ $\overline{\{n\}} \mathcal{M}^{\prime} / L$.

We need the following property to complete the proof:

(**) Let $M$ be a matroid on $[n]$ of rank $\geq 2$. For any two hyperplanes $H_{0}, H$ with $n \notin H_{0} \cup H$, there is a sequence of hyperplanes $H_{0}, H_{1}, \ldots, H_{k}=H$ such that $H_{i} \cap H_{i+1}$ is a coline, and $n \notin H_{i}, i=0, \ldots, k$.

Statement (**) is clear if the rank of $M$ is equal to 2 . Suppose $\operatorname{rank}(M)>2$. We use induction on the rank. If $\operatorname{rank}\left(H_{0} \cap H\right) \geq 1$, there exists a nonloop element $e \in H_{0} \cap H$ and the result is true in the contraction $M / e$ by the induction hypothesis and then in $M$. Suppose that $\operatorname{rank}\left(H_{0} \cap H\right)=0$, and consider a coline $L \subset H_{0}$. There is at least an element $a \in H \backslash L$ such that $n$ is not in the hyperplane $H^{\prime}:=\overline{L \cup\{a\}}$. Indeed, otherwise, $\bigcap_{a \in H \backslash L} \overline{L \cup\{a\}}$ must contain the hyperplane $\overline{L \cup\{n\}}$ and this is impossible because the former set is the intersection of at least two hyperplanes by the assumptions $\operatorname{rank}(M)>2$ and $\operatorname{rank}\left(H_{0} \cap H\right)=0$. Thus the property (**) follows from the first case for the pair $H^{\prime}$ and $H$. 
We consider now a hyperplane $H_{0}$ of $\underline{\mathcal{M}}$ such that $n \notin H_{0}$. Now, consider another hyperplane $H, n \notin H$. From (**) we know that there is a sequence of hyperplanes $H_{0}, H_{1}, \ldots, H_{m}=H$ such that $H_{i} \cap H_{i+1}$ is a coline, and $n \notin H_{i}, i=0, \ldots, m$. Hence, by property $\left(\mathrm{d}^{\prime}\right)$, all the cocircuits with $n$ in $\mathcal{M}$ and $\mathcal{M}^{\prime}$, are either equal, or all the cocircuits differ only in the sign of $n$. By Las Vergnas' single element extension theorem [12] (see also Proposition 7.1.4 of [2]), $\mathcal{M}=\mathcal{M}^{\prime}$ or $\mathcal{M}=\frac{}{\{n\}} \mathcal{M}^{\prime}$, and clearly $\mathcal{M}^{\prime} \in \mathrm{OC}(\mathcal{M})$.

The equivalence (a) $\Longleftrightarrow$ (d) of Theorem 2.3 is a generalization of Theorem 3.1 in [13] by duality. Also, in the rank 3 case, by identifying opposite points in the FolkmanLawrence representation in the sphere $S^{2}$, we obtain an arrangement of pseudolines in the real projective plane (see [10] for definitions); then the equivalence (a) $\Longleftrightarrow$ (b) reads as a theorem of Goodman and Pollack [8, Theorem 2.9].

An immediate consequence of the equivalence (a) $\Longleftrightarrow$ (d) of Theorem 2.3 is also:

Corollary 2.4 [5, Theorem 3.1]. Given an oriented matroid of rank $r$, for each $s, 2 \leq$ $s<r$, the labeled rank $s$ contractions determine the reorientation class of the oriented matroid.

We remark that Theorem 2.3 can be used to represent the reorientation class $\mathcal{M}$ in a simple graphic way: indeed, the vertex-labeled graph $\mathrm{G}_{\mathcal{M}}$ can be recovered from its decomposition in the circuits corresponding to the same labels of edges. Thus, in a way, this representation "generalizes" the notion of the Gale diagram [9], [16].

As a corollary, in the rank 3 case $G_{\mathcal{M}}$ determines by itself the isomorphism class of $\mathcal{M}$. More precisely, we have:

Corollary 2.5. Let $\mathcal{M}$ be a simple rank 3 oriented matroid. Then any of the following structures determine all the remaining:

(a) $\operatorname{IC}(\mathcal{M})$;

(b) the (unlabeled) cocircuit graph $\mathrm{G}_{\mathcal{M}}$ of $\mathcal{M}$;

(c) the (nonoriented) matroid $\underline{\mathcal{M}^{\prime}}$ for some $\mathcal{M}^{\prime} \in \operatorname{IC}(\mathcal{M})$, together with the class $\mathrm{OC}\left(\mathcal{M}^{\prime} / e\right)$ for every element $e \in E$ that is not a loop.

Proof. We prove that we may recover the structure in (c) of Theorem 2.3 from the one in (b) of the corollary, i.e., that we may read from $\mathrm{G}_{\mathcal{M}}$ a proper edge-labeling, which is uniquely determined up to isomorphism.

For this purpose, consider $\mathcal{W}$, the span of cocircuits of $\mathcal{M}^{\prime}$. By the Folkman-Lawrence representation theorem, there is an embedding of $\mathcal{W}$ into the sphere $S^{2}$. Since the cocircuit graph is 3-connected (in fact, it is 4-connected by Cordovil-Fukuda's theorem [6, Theorem 1.6]), the embedding is unique. All vertices of $\mathrm{G}_{\mathcal{M}^{\prime}}$ are of even degree, and the edges incident with the same vertex are circularly ordered in $S^{2}$. Since two such edges correspond to the same point of $\underline{\mathcal{M}^{\prime}}$ if and only if they are opposite in this ordering, the result follows now from Theorem 2.3. 


\section{Uniform Case}

In this section we consider the main theorem in the previous section, Theorem 2.3, in the special case when an oriented matroid is uniform. It turns out that only a little extra information besides the cocircuit graph determines the isomorphism class of a uniform oriented matroid.

Theorem 3.1. Let $\mathcal{M}$ be a uniform oriented matroid. Then the isomorphism class $\operatorname{IC}(\mathcal{M})$ of $\mathcal{M}$ is uniquely determined by the (unlabeled) cocircuit graph $\mathrm{G}_{\mathcal{M}}$ and the knowledge of the antipodal pairs of vertices in $\mathrm{G}_{\mathcal{M}}$ corresponding to the opposite pairs of cocircuits.

To prove this theorem, it is convenient to introduce a few definitions and a lemma. Let $M$ be a matroid of rank $r$. We denote by $\mathcal{L}=\mathcal{L}(M)$ the set of all colines. Define the distance $\operatorname{dist}(H, L)$ in $M$ from a hyperplane $H$ to a coline $L$ as the smallest number $s$ such that there exists a sequence of colines: $L_{0} \subseteq H, L_{1}, \ldots, L_{s}=L$ with $\operatorname{rank}\left(L_{i-1} \cup L_{i}\right)=$ $r-1$ for $i=1, \ldots, s$. We denote by $\mathcal{L}_{k}(H)$ the set of all colines whose distance from a hyperplane $H$ is $k$, for $k=0,1, \ldots$.

Lemma 3.2. Let $M$ be a uniform matroid and let $H$ be a hyperplane. Then

$$
\mathcal{L}_{k}(H)=\{L \subseteq E:|L|=r-2 \text { and }|L \backslash H|=k\} .
$$

Proof. This follows immediately from the uniformity.

Proof of Theorem 3.1. Again we prove that, besides permutation of the elements of $E$, we can read from $\mathrm{G}_{\mathcal{M}}$ its edge-labeling.

First, we prove that the edges of $\mathrm{G}_{\mathcal{M}}$ that correspond to the same coline of $\underline{\mathcal{M}}$ can be determined. By the uniformity, the rank $r$ is easily detected, i.e., $\delta=2(r-1)$ where $\delta$ is the degree of each vertex. The size $n$ of the ground set is still unknown but we explain below how one can recover it.

Let $P=\left[V=V_{0}, a_{0}, \ldots, a_{m}, V_{m+1}=-V\right]$ be a path in $\mathrm{G}_{\mathcal{M}}$ connecting the opposite vertices $V$ and $-V$. We assume for the moment that each vertex $V_{j}$ and each edge $a_{j}$ are both represented as sign vectors in the original matroid. It is easy to see that $m \geq n-(r-1)$, since $V$ and $-V$ have exactly $n-(r-1)$ nonzero components, and the number of (nonzero) components which are opposite in $V_{i-1}$ and $V_{i+1}$ is at most one for any $i=1, \ldots, m$. Hence the number of (nonzero) components which are opposite in $V_{0}$ and $V_{k}$ is at most $k-1$ for any $k=2, \ldots, m+1$. Furthermore, the path $P$ is a part of the graph $\mathrm{G}_{\mathcal{M} / L}$ for some coline $L$ if and only if $m=n-r+1$. The "only if" part is obvious. To show the "if" part, assume that the path is not a part of the graph $\mathrm{G}_{\mathcal{M} / L}$ for any coline $L$. This means that there are two distinct elements $e, f \in V^{0}$ and two vertices, say $V_{i}$ and $V_{j}$, such that $e \notin V_{i}^{0}$ and $f \notin V_{j}^{0}$. Since each of the $e$ and $f$ components of vertices must become nonzero and then zero independently in the path $P$, a simple count shows $m>n-r+1$.

Therefore, a path of minimum length between a pair of opposite vertices always corresponds to a coline, and the number $n$ can be determined from the length and $r$. In 
addition, two such paths correspond to the same coline if and only if they cross opposite vertices (in opposite ordering). Consequently we can partition the graph $\mathrm{G}_{\mathcal{M}}$ into an edge-disjoint union of cycles of length $2(n-r+2)$, each of which is the graph of a rank 2 contraction. We simply use "cycle" to mean these special cycles for the rest of the proof.

The only thing left to be shown is a construction of a proper label of each edge (or equivalently each cycle) by some $(r-2)$ subset of $[n]$, since Theorem 2.3 takes care of the rest.

Below we explain how each edge and each vertex of $G_{\mathcal{M}}$ can be labeled properly so that the resulting label is the labeling by an oriented matroid isomorphic to the original one.

First we select any vertex $V_{0}$ of $\mathrm{G}_{\mathcal{M}}$. By the freedom of isomorphism, one can label the vertex freely, and so we label it by $V_{0}=[r-1]$. Also there are exactly $(r-1)$ cycles containing $V_{0}$. We can label these cycles by the $(r-1)$ subsets of cardinal $(r-2)$ of $V_{0}$. Now we must use the remaining elements from $[n]$, i.e., $r, r+1, \ldots, n$. For this, we select any labeled cycle, say the one labeled by $[r-2]$. Half of this cycle is of the form $\left[V=V_{0}, a_{0}, \ldots, a_{m}, V_{m+1}=-V\right]$, where $m=n-r+1$. We can then label the intermediate vertices and their antipodes by $V_{i}=[r-2] \cup\{r+i-1\}$ for $i=1, \ldots, m$, since the remaining labels were never used before and must be used exactly once in this path.

Now the important claim is that all unlabeled vertices (and thus unlabeled edges) will get a unique label. This implies that the graph can be labeled without any ambiguity and thus properly. To prove this claim, we show how the labels can be assigned inductively. Let $H_{0}=V_{0}^{0}$ and

$$
\mathcal{L}_{k}\left(H_{0}\right)=\left\{L \subseteq[n]:|L|=r-2 \text { and }\left|L \backslash H_{0}\right|=k\right\}
$$

By Lemma 3.2, $\mathcal{L}_{k}\left(H_{0}\right)$ is the set of labels for the cycles of distance $k$ from $V_{0}$. The distance here is well defined since we have $G_{\mathcal{M}}$ and the adjacency of two cycles means they share a vertex. We denote by $\mathcal{C}_{k}$ the set of cycles in $\mathrm{G}_{\mathcal{M}}$ whose distance from $V_{0}$ is $k$.

All labels in $\mathcal{L}_{0}$ are already assigned to the cycles in $\mathcal{C}_{0}$, and the vertices of one cycle in $\mathcal{C}_{0}$ are assigned labels. The proof is completed by showing the two statements:

(a) The labels of vertices (of cycles) in $\mathcal{C}_{0}$ are uniquely determined by the labels already assigned.

(b) The labels of vertices and cycles in $\mathcal{C}_{k}$ are uniquely determined by the labels of vertices and cycles in $\mathcal{C}_{k-1}$, for any $k \geq 1$.

To prove (a), consider the vertex $V_{i}$ whose label is $[r-2] \cup\{r+i-1\}(i=1, \ldots, m)$. There are exactly $(r-2)$ cycles in $\mathcal{C}_{1}$ containing this vertex. Let $C$ be any one of them. It is easy to see that the cycle meets exactly two cycles of $\mathcal{C}_{0}$, one labeled by $[r-2]$ and the other by $[r-1] \backslash\{j\}$ for some $j \in[r-2]$. The antipodal pair of vertices in the intersection of these two cycles must get the label $[r-1] \backslash\{j\} \cup\{r+i-1\}$, and the cycle $C$ must be labeled $[r-2] \cup\{r+i-1\} \backslash\{j\}$. It is easy to see that every vertex on $\mathcal{C}_{0}$ will get a unique label this way. The proof of (b) is essentially the same, and we omit the details here. 
We conclude the paper by posing the following problem:

Problem 3.3. Does the (unlabeled) cocircuit graph $\mathrm{G}_{\mathcal{M}}$ of a uniform oriented matroid $\mathcal{M}$ determine its isomorphism class?

\section{References}

1. Björner, A.; Edelman, P. H.; Ziegler, G. M.: Hyperplane arrangements with a lattice of regions, Discrete Comput. Geom. 5(3) (1990), 263-288.

2. Björner, A.; Las Vergnas, M.; Sturmfels, B.; White, N.; Ziegler, G. M.: Oriented Matroids, Encyclopedia of Mathematics and its Applications 46, Cambridge University Press, Cambridge, 1993.

3. Bland, R. G.; Las Vergnas, M.: Orientability of matroids, J. Combin. Theory Ser. B 24(1) (1978), 94-123.

4. Blind, R.; Mani-Levitska, P.: Puzzles and polytope isomorphisms, Aequationes Math. 34(2-3) (1987), 287-297.

5. Bokowski, J.; Guedes de Oliveira, A.; Thiemann, U.; Veloso da Costa, A.: On the cube problem of Las Vergnas, Geom. Dedicata 63(1) (1996), 25-43.

6. Cordovil, R.; Fukuda, K.: Oriented matroids and combinatorial manifolds, European J. Combin. 14(1) (1993), 9-15.

7. Fukuda, K.; Saito, S.; Tamura, A.: Combinatorial face enumeration in arrangements and oriented matroids (First Canadian Conference on Geometry, Montreal, 1989), Discrete Appl. Math. 31(2) (1991), 141-149.

8. Goodman, J. E.; Pollack, R.: Semispaces of configurations, cell complexes of arrangements, J. Combin. Theory Ser. A 37(3) (1984), 257-293.

9. Grünbaum, B.: Convex Polytopes, Pure and Applied Mathematics 16, Wiley-Interscience, New York, 1967.

10. Grünbaum, B.: Arrangements and Spreads, Conference Board of the Mathematical Sciences Regional Conference Series in Mathematics 10, American Mathematical Society, Providence, RI, 1972.

11. Kalai, G.: A simple way to tell a simple polytope from its graph, J. Combin. Theory Ser. A 49(2) (1988), 381-383.

12. Las Vergnas, M.: Extensions ponctuelles d'une géométrie combinatoire orientée, in: Problèmes combinatoires et théorie des graphes (Colloq. Internat. CNRS, Univ. Orsay, Orsay, 1976), pp. 265-270, Colloq. Internat. CNRS 260, CNRS, Paris, 1978.

13. Roudneff, J.-P.: Reconstruction of the orientation class of an oriented matroid, European J. Combin. 9(5) (1988), 423-429.

14. Welsh, D. J. A.: Matroid Theory, L. M. S. Monographs, No. 8, Academic Press/Harcourt Brace Jovanovich, London/New York, 1976.

15. White, N. (ed.): Theory of Matroids, Encyclopedia of Mathematics and its Applications 26, Cambridge University Press, Cambridge, 1986.

16. Ziegler, G. M.: Lectures on Polytopes, Graduate Texts in Mathematics 152, Springer-Verlag, New York, 1994.

Received August 14, 1998, and in revised form March 2, 1999. Online publication May 3, 2000. 